BMJ

Open

Gastroenterology

\title{
Predictors of hepatic decompensation after TACE for hepatocellular carcinoma
}

\author{
Mohamed A S Kohla, ${ }^{1}$ Mai I Abu Zeid, ${ }^{1}$ Mohamed Al-Warraky, ${ }^{2}$ Hossam Taha, ${ }^{1}$ \\ Robert G Gish, ${ }^{3}$
}

\section{ABSTRACT}

Aim: To study predictive factors for hepatic decompensation after transarterial chemoembolisation (TACE) for hepatocellular carcinoma (HCC).

Methods: Between November 2009 and August 2010, of 254 patients with HCC who presented to our multidisciplinary HCC clinic for evaluation, 102 (40\%) were amenable for TACE. In this prospective study, there were 102 patients with compensated cirrhosis with HCC and Child-Pugh Class A cirrhosis who underwent TACE at the National Liver Institute, Menoufiya University, Egypt. We excluded all patients with prior locoregional therapy, systemic therapy and/or surgical intervention. At baseline and at 1 month postprocedure, laboratory criteria, tumour criteria (size, number) and Child-Pugh score were recorded. Patients were classified into group 1 (no ChildPugh point increase after TACE) and group 2 (one or more added Child-Pugh points after TACE, defining hepatic decompensation). Univariate and multivariate analyses were performed to identify factors predictive of hepatic decompensation.

Results: Patients were mostly males $(82.4 \%)$ of mean age $58.4 \pm 8.1$ years. The only significant changes in laboratory findings at 1 month after TACE were increased international normalised ratio, serum total bilirubin, alanine transaminase and aspartate transaminase and decreased serum albumin and $\alpha$-fetoprotein (AFP). The statistically significant predictive factors for hepatic decompensation using univariate analysis were found to be baseline lower serum albumin, higher serum $\alpha$-fetoprotein, more advanced Barcelona Clinic Liver Cancer (BCLC) stage, larger tumour size and a greater number of tumour nodules; with logistic regression, multivariate analysis found that at baseline larger tumour size $(p=0.004$ at $95 \% \mathrm{Cl})$, higher serum AFP $(p=0.046$ at $95 \% \mathrm{Cl})$ and lower serum albumin $(p=0.033$ at $95 \% \mathrm{Cl})$ predicted decompensation; BCLC stage, number of tumour nodules and pre-TACE bilirubin did not predict changes in liver function.

Conclusions: Lower serum albumin and increased tumour burden (larger tumour size/more nodules and higher $\alpha$-fetoprotein) at baseline may help predict postTACE decompensation.

\section{INTRODUCTION}

Transarterial chemoembolisation (TACE) has become the most popular modality for

\section{Summary box}

What is already known about this subject?

- Transarterial chemoembolisation (TACE) or transarterial bead embolisation (TABE) is the standard of care for management of patients with intermediate stage (Barcelona Clinic Liver Cancer (BCLC) stage B) hepatocellular carcinoma (HCC).

- TACE was found to improve survival compared with supportive care in patients with unresectable HCC.

- Hepatic insufficiency is the most common adverse effect after TACE.

What are the new findings?

- Unlike most studies addressing predictive factors for decompensation after TACE which were retrospective, we carried out a prospective study which found that serum albumin and tumour burden (tumour size and $\alpha$-fetoprotein (AFP)) were the most predictive factors for hepatic decompensation after TACE.

- This study is unique in that all patients had undergone the same technique of TACE in the same centre and were all assessed after one session of TACE to minimise bias.

- This study provides simple predictive variables that are easy to use by clinicians.

How might it impact on clinical practice in the foreseeable future?

- If future prospective studies on larger numbers of patients confirm the predictive value of these two variables for post-TACE hepatic decompensation, a cut-off value for serum albumin and serum AFP could be determined which could help identify patients at high versus low risk for liver decompensation.

- These findings could be of significant help to identify at-risk patients who are more prone to develop hepatic decompensation after TACE and to assist treating physicians in planning treatment strategies for individual patients as part of personalised medicine.

palliative treatment among patients with hepatocellular carcinoma (HCC). ${ }^{1}{ }^{2}$ TACE may slow tumour progression and improve survival by combining the effect of targeted chemotherapy with that of ischaemic 
necrosis induced by arterial embolisation. TACE was found to improve survival compared with supportive care in patients with unresectable HCC. ${ }^{3}$ Hepatic artery obstruction, known as transarterial or transcatheter arterial embolisation (TAE), is performed during an angiographic procedure. When TAE is combined with prior injection into the hepatic artery of chemotherapeutic agents, usually mixed with ethiodised oil (ethiodol, lipiodol), the procedure is known as TACE. ${ }^{4-6}$ Transarterial bead embolisation (TABE) is also used at some centres.

Several studies have focused on the impact of TACE for the treatment or palliation of unresectable HCC. In two randomised controlled trials and one systematic review with meta-analysis, TACE was found to improve survival compared with supportive care in patients with unresectable HCC. ${ }^{3}$ According to the guidelines published by the American Association for the Study of Liver Diseases $(\mathrm{AASLD})^{5}$ and the European Association for the Study of the Liver (EASL), ${ }^{7}$ TACE is recommended as a first-line non-curative therapy for non-surgical patients with large/ multifocal HCC who do not have a vascular invasion or an extrahepatic spread. Exclusion criteria in most trials were advanced liver disease (Child-Pugh C), active gastrointestinal bleeding, encephalopathy, refractory ascites, presence of vascular invasion due to liver tumour, extrahepatic metastases, portosystemic shunt, hepatofugal blood flow, any contraindication to an arterial procedure (impaired clotting tests and renal failure), Eastern Cooperative Oncology Group (ECOG) performance stage 3 or 4 and end-stage tumorous disease. ${ }^{8}$

The prognosis of patients with HCC can best be assessed by taking tumour stage, liver function and physical status into account in the staging system; this may also potentially determine who will be stable and who will decompensate after TACE. The impact of treatment should also be considered when starting HCC treatment and when estimating life expectancy. Currently, the Barcelona Clinic Liver Cancer (BCLC) staging system is the only staging system that accomplishes these aims. ${ }^{9}$ Although TACE has marked direct antitumour effects, it can also result in more complications than conservative management. Signs of liver injury and hepatic insufficiency are the most common adverse effects after TACE, with elevated aminotransferases and negative changes in liver function tests seen in almost all patients. ${ }^{10-13}$ Studies suggest that the elevations of aminotransferases after TACE result from not only the ischaemic or hypoxic damage of non-tumorous liver but also from the tumour necrosis caused by TACE. Some of the elevation may also be due to the enzyme release from lytic hepatoma cells after TACE. ${ }^{14}{ }^{15}$ The benefits of TACE can be offset by treatment-induced liver deterioration to the detriment of patient care. Predictors of poor patient outcome in the published literature are related to tumour burden (tumour size, vascular invasion and $\alpha$-fetoprotein(AFP) levels), liver functional impairment (Child-Pugh score, serum bilirubin, ascites), health status (constitutional syndrome, performance status (PST)) and response to treatment. ${ }^{9}$ We prospectively assessed patient and tumour characteristics and laboratory findings in order to determine which factors might best predict post-TACE hepatic decompensation.

\section{MATERIALS AND METHODS}

From November 2009 to August 2010, a total of 254 patients with HCC presented to our multidisciplinary HCC clinic for evaluation. The underlying liver disease was hepatitis $\mathrm{C}$ virus-induced cirrhosis in 216 patients $(85 \%)$, chronic hepatitis B in 25 patients $(9.9 \%)$ and non-alcoholic steatohepatitis in 13 patients $(5.1 \%)$. Twenty-six patients $(10.2 \%)$ were amenable to surgical management (14 had living donor liver transplantation and 10 had resection); 46 patients $(18 \%)$ were amenable to local ablation (30 had radiofrequency ablation and 16 had microwave ablation); 102 patients $(40 \%)$ had TACE; and 80 patients $(31.4 \%)$ were treated by best supportive care (BSC). The treatment decision was made according to the BCLC staging algorithm. Sorafenib is not routinely used in Egypt due to its high cost and economic burden. Thus, most patients with advanced stage HCC (BCLC stage $\mathrm{C}$ ) were treated by BSC; only a small proportion of patients with BCLC stage $\mathrm{C}$ with very good hepatic reserve were treated with TACE as a palliative measure. This prospective study included 102 compensated patients with cirrhosis with hepatocellular carcinoma and Child-Pugh Class A cirrhosis who underwent TACE. Excluded were all patients with prior locoregional therapy (including those having multiple TACE treatments), systemic therapy and/or surgical intervention (liver resection or orthotopic liver transplantation). All patients enrolled in this study (102 patients) gave their informed written consent prior to their inclusion in the study. The study was revised and approved by the local ethical committee and institutional review board (IRB) of the National Liver Institute.

\section{Data collection}

At baseline and at 1 month post-TACE, demographic information, laboratory measurements, radiological tumour assessments and clinical information were recorded (tables 1 and 2). Radiological assessment was performed using quad-phasic multidetector CT (Siemens definition A, 20-slices) with non-contrast, arterial, portal and delayed phases. The tumour assessments included the number of nodules, unilobar versus bilobar, nodule size if single and total tumour volume if multinodular (sum of the sizes of all tumours). All quad-phasic CT images were assessed by the same radiologist. According to the Common Terminology Criteria for Adverse Events V.3.0 (CTACE), it is recommended to assess TACE-related adverse events 4 weeks after the procedure. ${ }^{16}$ Moreover, in this study, we evaluated patients 1 month after TACE because (1) in our practice, all patients are evaluated at this time after TACE by a four phase CT to assess whether the patient needed a 


\section{Table 1 Demographics, tumour characteristics and BCLC staging}

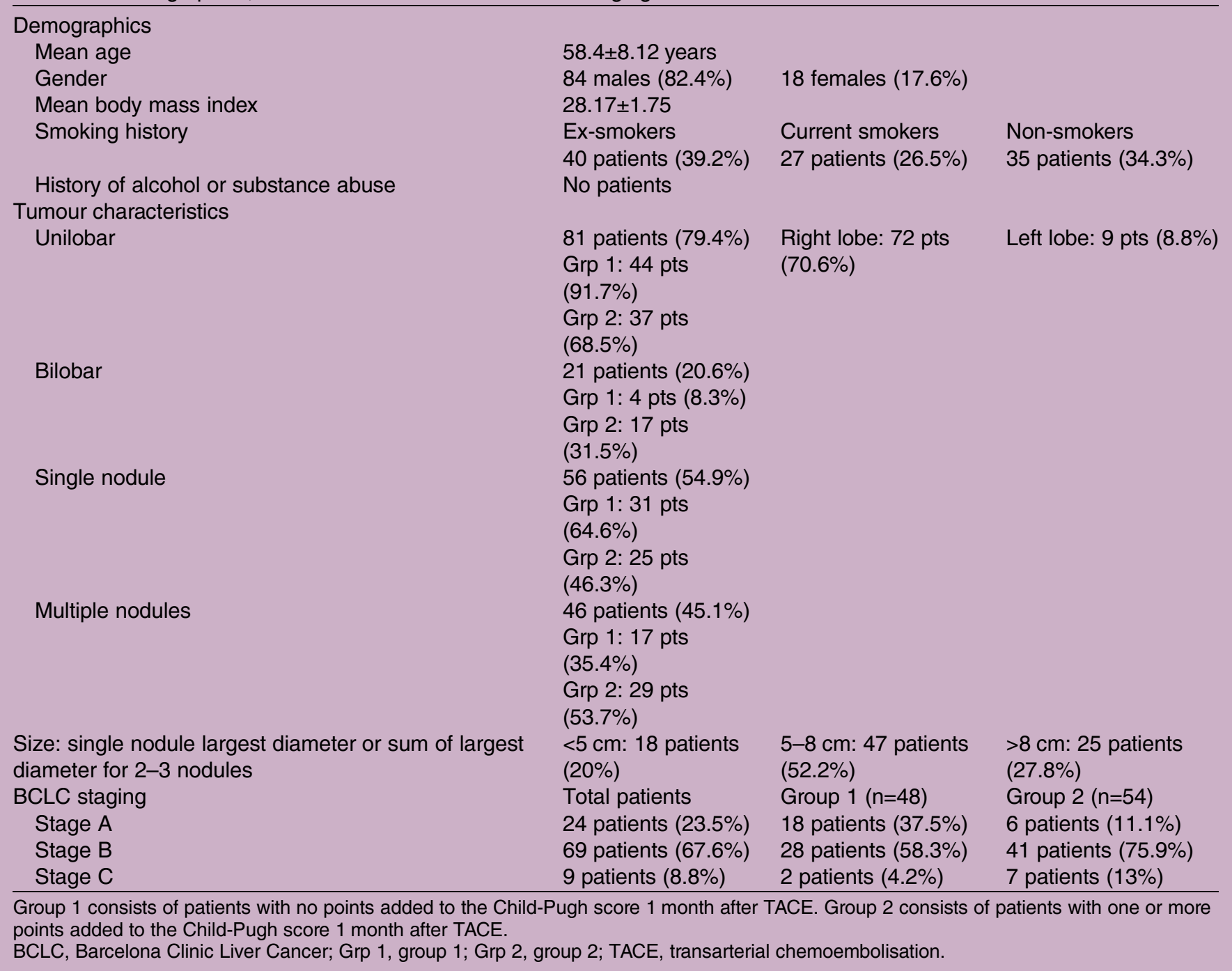

second treatment session; and (2) long-term decompensation is more clinically relevant than transient decompensation after TACE because it might affect a subsequent treatment strategy following TACE both at 1 month and at longer assessment intervals.

\section{Transarterial chemoembolisation}

Chemoembolisation was performed percutaneously at the angiography unit of the National Liver Institute with the patient under conscious sedation. After infiltration of a local analgesic, the Seldinger technique was used to gain access to the common femoral artery through a femoral artery puncture. A 5-french vascular sheath was placed into the common femoral artery over a 0.035-inch guide wire. Under fluoroscopic guidance, a 5-french glide Cobracatheter (Cordis) was advanced into the aorta. An angiographic study of the superior mesenteric artery (SMA), coeliac trunk and common hepatic artery was performed to identify all of the vessels feeding the HCC nodule and to assess the patency of the portal vein. In some patients, selective angiography of the phrenic or intercostal arterial branches was required. The arterial branches feeding the tumour are selectively cannulated by microcatheters to proceed with TACE and to ensure better preservation of the surrounding non-tumoral liver tissue. An emulsion of lipiodoldoxorubicin $(50 \mathrm{mg}$ of doxorubicin was mixed with 6-20 mL of lipiodol according to the tumour size, number and vascularity to form the emulsion) was injected; the injection was performed far from the origin of the gastroduodenal, right gastric and cystic arteries; the amount injected into the tumour was adjusted according to the size and uptake of the tumour. Gel foam was the embolic material injected in all patients.

\section{Statistical analysis}

Data were collected and entered using the SPSS (Statistical Package for the Social Sciences) program for statistical analysis, (V.13; IBM Corporation, Armonk, New York). Laboratory findings (before and after TACE) were expressed as the mean and SD $(\mathrm{X} \pm \mathrm{SD})$ and analysed by applying paired t test for comparison of normally distributed variables and Wilcoxon signed ranks test for non-normally distributed ones. 
Table 2 One month post-TACE: laboratory findings, clinical manifestations of hepatic decompensation, and changes in the Child-Pugh score

\begin{tabular}{|c|c|c|c|}
\hline Mean laboratory findings & Baseline & 1 month post-TACE & $\begin{array}{l}\text { Significant change? } \\
\text { ( } p \text { values at } 95 \% \mathrm{Cl} \text { ) }\end{array}$ \\
\hline Haemoglobin & $12.36 \pm 1.66$ & $12.31 \pm 1.73$ & No $(p=0.16)$ \\
\hline Platelet count & $147.7 \pm 64.85 \times 10^{3} / \mathrm{L}$ & $137.86 \pm 67.46 \times 10^{3} / \mathrm{L}$ & No $(p=0.06)$ \\
\hline INR & $1.17 \pm 0.13$ & $1.28 \pm 0.26$ & Yes $(p=0.008)$ \\
\hline Serum total bilirubin & $1.09 \pm 0.46$ & $1.63 \pm 1.01$ & Yes $(p<0.001)$ \\
\hline Serum albumin & $3.54 \pm 0.45$ & $3.1 \pm 0.56$ & Yes $(p<0.001)$ \\
\hline AST & $71 \pm 33$ & $85 \pm 42$ & Yes $(p<0.001)$ \\
\hline ALT & $57 \pm 27$ & $67 \pm 29$ & Yes $(p<0.001)$ \\
\hline AFP & $1056.79 \pm 2057.59$ & $794.53 \pm 1578.62$ & Yes $(p<0.001)$ \\
\hline Blood urea & $31.27 \pm 7.58$ & $30.73 \pm 6.59$ & No $(p=0.19)$ \\
\hline Serum creatinine & $0.81 \pm 0.19$ & $0.83 \pm 0.22$ & No $(p=0.32)$ \\
\hline Sodium & $136.97 \pm 4.01$ & $136.81 \pm 3.75$ & No $(p=0.70)$ \\
\hline Potassium & $4.2 \pm 0.49$ & $4.13 \pm 0.51$ & No $(p=0.19)$ \\
\hline Clinical manifestations & Total patients & Group 1 patients & Group 2 patients \\
\hline Haematemesis & $10(9.8 \%)$ & 1 of 48 pts $(2.1 \%)$ & 9 of 54 pts $(16.7 \%)$ \\
\hline Hepatic encephalopathy & $9(8.8 \%)$ & None & 9 of 54 pts $(16.7 \%)$ \\
\hline Ascites & $25(24.5 \%)$ & None & 25 of 54 pts $(46.3 \%)$ \\
\hline Child-Pugh score & Baseline & 1 month post-TACE & \\
\hline A & 102 pts (100\%) & 60 pts $(58.8 \%)$ & \\
\hline \multirow[t]{3}{*}{ A5 } & 50 pts $(49 \%)$ & & \\
\hline & $\begin{array}{l}\text { Grp 1: } 28 \text { pts } \\
(58.3 \%)\end{array}$ & & \\
\hline & Grp 2: 23 pts & & \\
\hline \multirow[t]{3}{*}{ A6 } & $(42.5 \%)$ & & \\
\hline & 52 pts $(51 \%)$ & & \\
\hline & Grp 1: 20 pts & & \\
\hline B & $(41.6 \%)$ & 31 pts $(30.4 \%)$ & \\
\hline \multirow[t]{3}{*}{ C } & $\begin{array}{l}\text { Grp 2: } 31 \text { pts } \\
(57.4 \%)\end{array}$ & 11 pts $(10.8 \%)$ & \\
\hline & None & & \\
\hline & None & & \\
\hline
\end{tabular}

Quantitative data for each group were expressed as the mean and $\mathrm{SD}(\mathrm{X} \pm \mathrm{SD})$ and univariate analysis was performed by applying student $t$ test for comparison of the two groups for normally distributed variables and Mann-Whitney $U$ test for non-normally distributed ones. Qualitative data were expressed as the number and percentage and univariate analysis was performed by applying $\chi^{2}$ test $\left(\chi^{2}\right)$ for comparison of the two groups. Multivariate analysis was performed using the binary logistic regression model. Significance was established as $\mathrm{p}<0.05$.

\section{RESULTS}

\section{Demographics}

Patients were mostly males $(82.4 \%)$ of mean age 58.4 \pm 8.12 years (see table 1 for complete demographics).

\section{Laboratory findings}

The only significant changes in laboratory findings at 1 month after TACE were increased international normalised ratio (INR), serum total bilirubin, alanine transaminase and aspartate transaminase, and decreased serum albumin and AFP. There was no significant change in haemoglobin, platelet count, serum electrolytes or measures related to renal function (See table 2 for full details on laboratory findings).

\section{Tumour characteristics and BCLC staging}

Most patients (79.4\%) had unilobar tumours, most of which $(70.6 \%)$ were in the right lobe. Most of the patients $(54.9 \%)$ had a single nodule; $27.5 \%$ had 2 nodules; $5.9 \%$ had 3 nodules; and $11.8 \%$ had $>3$ nodules. The nodule size (largest diameter of a single nodule or sum of the largest diameter for 2-3 nodules) equalled $<5 \mathrm{~cm}$ in 18 patients $(20 \%) ; 5-8 \mathrm{~cm}$ in 47 patients $(52.2 \%)$; and $>8 \mathrm{~cm}$ in 25 patients $(27.8 \%$; See table 1 for full information on tumour characteristics). Twenty-four patients $(23.5 \%)$ were BCLC stage A; 69 patients $(67.6 \%)$ were stage $\mathrm{B}$; and 9 patients $(8.8 \%)$ were stage $\mathrm{C}$ (table 1 ). 
Decompensation defined by changes in the Child-Pugh score 1 month after TACE

All the 102 patients had a baseline Child-Pugh score A, $50(49 \%)$ with a score of A5 and $52(51 \%)$ with a score of A6. One month after TACE, 60 patients out of 102 $(58.8 \%)$ remained at Child-Pugh score A, while 31 patients $(30.4 \%)$ became Child-Pugh score B and 11 patients $(10.8 \%$ ) became Child-Pugh score C (table 2). Patients were divided into group 1, 48 patients $(47.1 \%)$ with no points added to the Child-Pugh score 1 month after TACE, and group 2, 54 patients $(52.9 \%)$ with one or more points added. One month post-TACE, the mean serum bilirubin and INR were significantly higher and mean serum albumin was significantly lower in group 2 $(\mathrm{p}<0.001$ at $95 \%$ CI ) (table 3). Superselective embolisation, which may reflect a lower risk of hepatic deterioration in large and multiple tumours, was performed for 17 out of 48 patients in group $1(35.4 \%)$, versus 29 out of 54 patients in group $2(53.7 \%)$, with a $\mathrm{p}$ value of 0.064 which was not statistically significant.

None of the patients in the two groups developed any serious adverse events specific to the TACE procedure (eg, local bleeding, abscess formation). Only one patient from group $1(2.1 \%)$ developed haematemesis 1 month after TACE versus 9 patients from group 2 (16.7\%), reflecting a significant difference $(\mathrm{p}=0.01$ at $95 \% \mathrm{CI}$; table 2). No one from group 1 developed hepatic encephalopathy or ascites 1 month after TACE, while 9 patients (16.7\%) from group 2 developed hepatic encephalopathy and 25 patients (44.4\%) developed ascites (table 2).

\section{Changes at ECOG performance status}

All patients in both groups had an ECOG-PS of 0 or 1 before the procedure. After TACE, 3 out of 48 patients in group $1(6.25 \%)$ had an increase in their ECOG-PS to $>1$, versus 10 out of 54 patients in group $2(18.5 \%)$, with a $\mathrm{p}$ value of 0.64 which was not statistically significant.

\section{Survival analysis}

The median overall survival for all patients in the two groups (102 patients) was 19 months.

Figure 1 shows a Kaplan-Meier survival analysis for all patients in the two groups.

Table 3 Comparison between group 1 and group 2 of laboratory tests that reflect hepatic reserve 1 month after TACE

\begin{tabular}{lllll}
\hline & $\begin{array}{l}\text { Group 1 } \\
(\mathbf{N}=48)\end{array}$ & $\begin{array}{l}\text { Group 2 } \\
\mathbf{( N = 5 4 )}\end{array}$ & & \\
$\mathbf{X + S D}$ & $\mathbf{X + S D}$ & t test & p Value \\
\hline Bilirubin & $1.09 \pm 0.31$ & $2.12 \pm 1.16$ & $5.12^{*}$ & $<0.001$ \\
INR & $1.16 \pm 0.5$ & $1.38 \pm 0.25$ & 5.45 & $<0.001$ \\
Albumin & $3.46 \pm 0.42$ & $2.78 \pm 0.47$ & 7.7 & $<0.001$ \\
\hline *Mann whitney test. & & & \\
TACE, transarterial chemoembolisation.
\end{tabular}

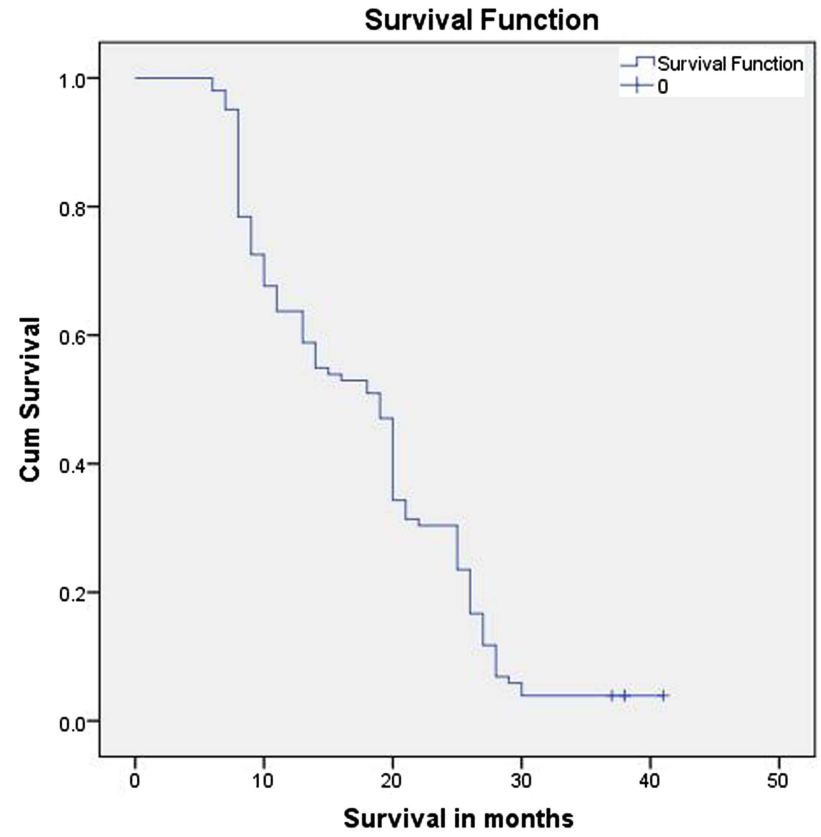

Figure 1 Overall survival for all patients. Kaplan-Meier survival analysis for all patients in the two groups.

After 1 year of follow-up, 41 out of 48 patients in group $1(84.5 \%)$ were alive, versus 24 out of 54 patients in group $2(44.4 \%)$, with a $\mathrm{p}$ value $<0.01$ which was statistically significant.

After 2 years of follow-up, 21 out of 48 patients in group $1(43.75 \%)$ were alive, versus 10 out of 54 patients in group $2(18.5 \%)$, with a $\mathrm{p}$ value $<0.01$ which was statistically significant.

After 3 years of follow-up, 4 out of 48 patients in group $1(8.3 \%)$ were alive, while all patients in group 2 were deceased, with a $\mathrm{p}$ value $<0.05$ which was statistically significant. Patients in group 1 had a median survival of 21 months, which was significantly higher than that for patients in group 2, who had a median survival of 10 months, with a $p$ value $<0.001$.

Figure 2 shows a Kaplan-Meier survival analysis of group 1 versus group 2.

\section{Calculation of the STATE score}

The STATE score was calculated for all patients; it was found that 12 out of 48 patients in group $1(25 \%)$ had a STATE score $<18$, versus 26 out of 54 patients in group 2 $(48 \%)$, with a $\mathrm{p}$ value of 0.016 which was statistically significant.

\section{Survival analysis according to the STATE score}

In our cohort, 64 patients $(62.7 \%)$ had a STATE score $\geq 18$, and 38 patients $(37.3 \%)$ had a STATE score $<18$. Patients with a STATE score $\geq 18$ had a median survival of 21 months, while those with a STATE score $<18$ had a median survival of 9 months; the difference was highly significant with a $\mathrm{p}$ value $<0.001$. Figure 3 shows a 


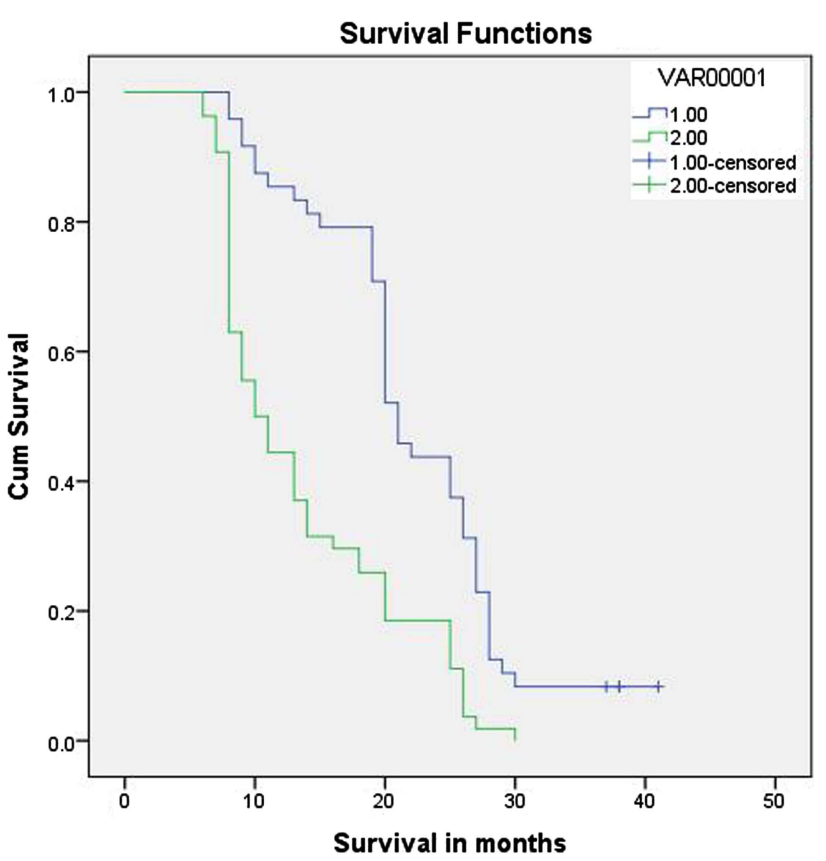

Figure 2 Survival of group 1 versus group 2. Kaplan-Meier survival analysis of group 1 (blue legend), versus group 2 (green legend).

Kaplan-Meier survival analysis of patients with a STATE score $\geq 18$, versus those with a STATE score $<18$.

\section{Calculation of the HAP score}

The HAP score was calculated for all patients in the 2 groups. In group 1 (48 patients), 10 patients were HAP A, 15 were HAP B, 17 were HAP C and 6 were HAP D,

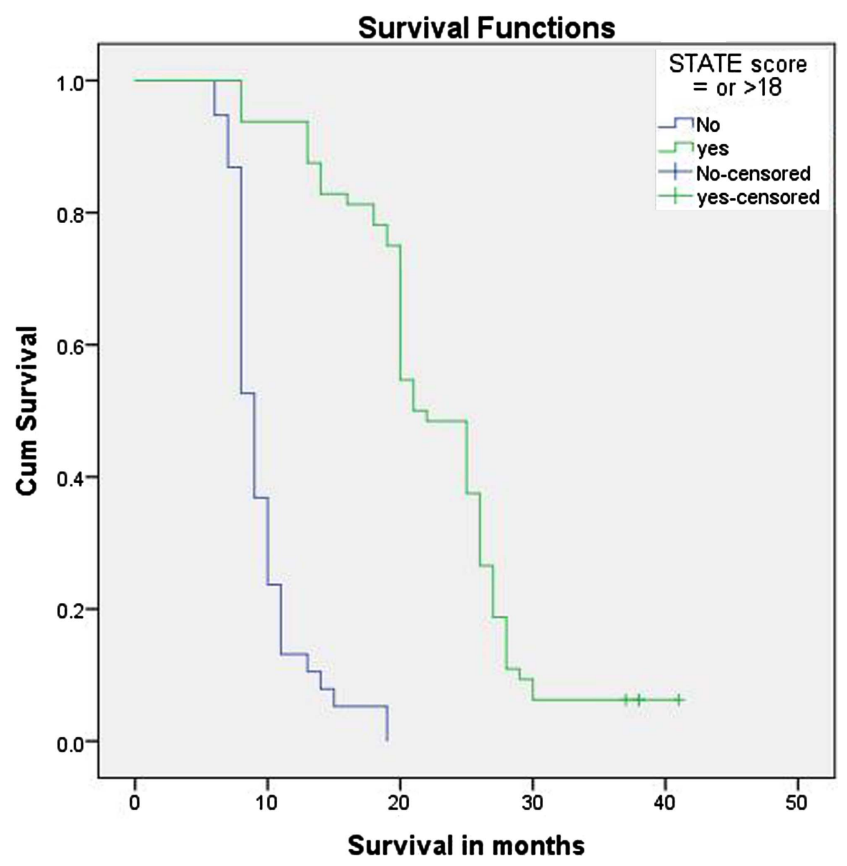

Figure 3 Survival of patients with a STATE score $\geq 18$ versus patients with a STATE score $<18$. Kaplan-Meier survival analysis of patients with a STATE score $\geq 18$ (green legend), versus those with a STATE score $<18$ (blue legend). while in group 2, 8 were HAP A, 13 were HAP B, 21 were HAP C, and 12 were HAP D. Overall, 23 out of 48 patients in group $1(47.9 \%)$ had a more advanced HAP score (HAP C and D), versus 33 out of 54 patients in group $2(61.1 \%)$, with a $\mathrm{p}$ value of 0.181 which was not statistically significant.

\section{Univariate analysis for variables predictive for hepatic decompensation}

Univariate analysis was performed to compare the two groups for the different preprocedure variables that might predict decompensation postprocedure. There was no significant difference between group 1 and group 2 as regards gender, age, body mass index (BMI) or smoking. There was no significant difference in baseline laboratory findings between the two groups other than baseline serum albumin, which was significantly lower in group $2(\mathrm{p}=0.012$ at $95 \% \mathrm{CI})$, and baseline serum AFP, which was significantly higher in group 2 ( $p=0.02$ at $95 \%$ CI). There was no significant difference in the baseline Child-Pugh scores between group 1 and group 2 (table 2). As regards BCLC staging, there was a statistically significant difference $(p=0.005$ at $95 \%$ CI $)$ between the two groups showing that patients in group 2 were of more advanced BCLC (table 1).

As shown in table 1, the number of patients with bilobar tumours was significantly higher in group 2, while the number of patients with unilobar tumours was significantly higher in group $1(\mathrm{p}=0.004$ at $95 \% \mathrm{CI})$; the number of patients with more than one nodule was significantly higher in group $2(\mathrm{p}=0.04$ at $95 \% \mathrm{CI})$. There was no statistically significant difference between the two groups as regards vascular invasion and extrahepatic spread.

From the above univariate analysis, serum albumin, AFP, BCLC staging and site, number and size of tumour nodules were the statistically significant factors predictive of decompensation. A stepwise binary logistic regression multivariate analysis was run, revealing that the largest tumour diameter $(\mathrm{p}=0.004$ at $95 \%$ CI $)$, serum AFP ( $p$ value $=0.046$ at $95 \%$ CI) and serum albumin $(p=0.033$ at $95 \% \mathrm{CI}$ ) were the statistically significant independent factors predictive of decompensation after TACE with ORs of 4.45, 1.12 and 0.31 , respectively (table 4 ).

\section{DISCUSSION}

TACE can result in more complications than conservative management. However, most published studies of TACE to date have focused on patient survival rather than hepatic safety. In the few studies on hepatic safety, the number of cases is low and the identified complications are not inclusive. ${ }^{12} 17$ Only a few studies have evaluated the effects of TACE on hepatic function. ${ }^{2} \quad 8 \quad 18 \quad 19$ Data related to TACE-induced liver decompensation are difficult to compare because of the different definitions used in each study. Liver decompensation has been variously defined by the occurrence of any of the following: 
Table 4 Stepwise logistic regression multivariate analysis of the statistically significant variables of the univariate analysis

\begin{tabular}{llll}
\hline Variable & OR & 95\% Cl & p Value \\
\hline Size of nodules & 4.45 & $(1.63$ to 8.25$)$ & 0.004 \\
AFP & 1.12 & $(1.1$ to 1.3$)$ & 0.046 \\
Serum albumin & 0.31 & $(0.11$ to 0.91$)$ & 0.033 \\
BCLC & 2.75 & $(0.43$ to 3.92$)$ & 0.64 \\
Site of nodules & 1.54 & $(0.12$ to 2.60$)$ & 0.44 \\
Number of nodules & 1.227 & $(0.425$ to 2.74$)$ & 0.79 \\
\hline \multicolumn{4}{l}{ BCLC, Barcelona Clinic Liver Cancer; AFP, $\alpha$-fetoprotein. }
\end{tabular}

encephalopathy, increasing ascites, increase in prothrombin time, increase in the serum bilirubin and/or deterioration of the Child-Pugh status. ${ }^{20}$ Unlike most published studies to date, this manuscript provides the results of a prospective study. The TACE technique was the same and was constant in all patients; none of the patients received any specific treatment for HCC prior to TACE which minimises bias in data interpretation and conclusions. Evaluation of hepatic decompensation after one session of TACE eliminates the confounding effect of multiple TACE sessions on hepatic reserve, which was the case in most of the other TACE outcome studies. However, heterogeneity of the study cohort is a limitation in many of the TACE studies because of the wide spectrum of patients with HCC eligible for TACE compared to other modalities of treatment of HCC. This can be overcome by conducting more prospective studies on a larger number of patients.

According to the 2010 AASLD practice guidelines, TACE is only indicated for intermediate stage HCC. ${ }^{5}$ The majority of our patients were BCLC stage B. For those with BCLC stage A, there are curative treatment modalities such as surgery or radiofrequency ablation. TACE was performed for our patients either because they had a contraindication to other treatment options or as a bridge until there was a facility for curative treatment options. It has been shown that TACE can be used as a bridging therapy to control tumour growth and achieve significant tumour necrosis, which may reduce tumour dissemination during surgery. ${ }^{9}$ Only a few of our cases were BCLC stage C. Although TACE is usually not performed for this stage of the disease because liver vascular invasion and/or extrahepatic spread are contraindications, some studies have indicated that TACE is safe even in those with complete or partial thrombosis of the portal vein. ${ }^{21-24}$ Even though the danger of provoking acute liver failure is estimated to be quite small if liver function is well preserved, ${ }^{25}$ there are concerns about the long-term consequences of hepatic function. ${ }^{24}$ Herber $e t a l^{25}$ concluded that the presence of portal vein thrombosis at the initial diagnosis of HCC is not an absolute contraindication for TACE treatment but that patients must be selected carefully with critical regard to their liver function. A recent study showed that TACE can be performed safely and may improve the overall survival of patients with HCC and main portal vein invasion. ${ }^{26}$ The presence of extrahepatic metastases is a relative contraindication for TACE. Some physicians use TACE to control the growth of intrahepatic tumours even in the presence of an extrahepatic spread, ${ }^{27}$ but the actual clinical benefit is variable. ${ }^{28}$

In our study, there was no significant change in CBC, renal function or serum electrolytes. Although a fall in platelet count after TACE (generally due to an increase in portal hypertension, drug toxicity or both) has been observed by a number of other authors, ${ }^{24} 29-32$ in our study, the reduction in platelet count did not reach statistical significance. As shown by the significantly reduced mean serum albumin and increased INR, there was a substantial impact on synthetic liver function as well as on serum AFP. We also found that TACE induced a significant hepatic ischaemia as shown by the elevated AST and ALT levels. These post-TACE aminotransferase elevations result from not only the ischaemic damage, but also from the tumour necrosis caused by TACE. ${ }^{15} 33$ Sun et $a l^{12}$ reported that cirrhotic livers are more sensitive to the damage of ischaemia. Herber $e t a l^{24}$ also found that liver function worsened significantly in their cohort. Gu et $a \hat{l}^{34}$ reported that 10 of 15 patients experienced liver dysfunction, and that the mean levels of ALT and AST rose to 600-1200 U/L in 'serious' cases (greater than a 10-fold increase in transaminases). Other studies have reported that although deterioration of liver function recovers to the pretreatment level before the next session of TACE in most patients, some have had irreversible hepatic decompensation. ${ }^{20} 35$

The Selection for TrAnsarterial chemoembolisation TrEatment (STATE) score was recently developed to guide the decision for the first TACE treatment in patients with HCC. ${ }^{36}$ Based on the impact of baseline liver function and tumour load on overall survival, the STATE score starts with the serum albumin level $(\mathrm{g} / \mathrm{dL})$, which is reduced by 12 points each if the tumour load exceeds the up-to-7 criteria and/or $\mathrm{C}$ reactive protein (CRP) levels are $\geq 1 \mathrm{mg}$ / $\mathrm{dL}$ (maximum reduction: 24 points). In a retrospective analysis, it was shown to identify patients who are unsuitable for TACE, with a lower STATE score associated with increased mortality post-TACE. The STATE score was validated in our cohort which differs from the HCC cohort in western Europe (most HCC cases in Egypt develop on top of HCV-induced cirrhosis); this validation in a different cohort of patients is clinically significant.

Another prognostic score, the Hepatoma Arterial embolization Prognostic (HAP) score, was recently developed. ${ }^{37}$ The HAP score is based on serum albumin, serum bilirubin, AFP and dominant tumour size and was shown to predict the outcome in patients with $\mathrm{HCC}$ undergoing TACE/TAE. Unlike the STATE score, the HAP score did not show a significant difference when comparing the two groups in our study. This could be attributed to the relatively smaller number of patients in our study. We believe that validation of the HAP score will require studies of larger numbers of patients. AFP is 
a low quality risk marker for cancer development when used alone. It is an inaccurate test to stage HCC and is also a poor prognostic test for HCC complications as a binary test. Thus, large studies are needed to refine the use of AFP as a single test or as part of a composite analysis to improve the sensitivity or accuracy of scores such as the HAP score.

Our finding of significantly reduced serum AFP indicates that TACE may have significantly reduced tumour burden. The level of AFP attributed to extensive tumour necrosis has been reported to be correlated with tumour burden in previous studies. ${ }^{35}$ 38-40 A hypervascular tumour may be successfully devascularised with a dramatic fall in AFP and change in imaging on arterial and venous phases, but the size of the tumour may remain unchanged. ${ }^{20} \mathrm{~A}$ recent meta-analysis showed an improvement in the overall survival of patients with wellpreserved liver function who were given intra-arterial treatment. ${ }^{41}$ There was no TACE-related mortality in our study and the cause of death in most cases was related to disease progression. The reason for this may be that all of our patients had compensated (Child-Pugh Class A) cirrhosis with preserved liver function and that none of the cases with vascular invasion had complete main portal vein thrombosis.

With our division of patients into a group with no points added to their baseline Child-Pugh score 1 month after TACE (group 1) and a group with one or more points added (group 2), we were able to confirm that group 2 patients are those who had some degree of hepatic decompensation 1 month after TACE. By comparing the laboratory tests that reflect hepatic reserve, it was obvious that the group 2 patients had a significantly worse reserve. In addition, we found that all patients who presented with clinical manifestations of hepatic decompensation 1 month after TACE belonged to group 2 , with the exception of only one patient from group 1 who developed haematemesis for which portal hypertension was the main factor predicting gastrointestinal bleeding. Univariate analysis of the two groups' baseline laboratory findings showed that the only significant differences were significantly lower serum albumin and significantly higher serum AFP in group 2 patients. These also proved to be independent predictive factors for decompensation on multivariate analysis. Since serum albumin is one of the most important factors in evaluating hepatic synthetic function, ${ }^{39} 42$ it was included in the Okuda staging system for $\mathrm{HCC}^{43}$ and Child-Pugh classification. It was reported that liver function deterioration after TACE is more common in patients with less liver reserve $^{10} 244^{44-46}$ Higher AFP levels may reflect a more advanced stage and greater malignant potential of tumours which may correlate with liver function changes after TACE. ${ }^{35}$ Several studies have revealed its value as an independent prognostic factor associated with poor outcome. ${ }^{24} 424447$

Regarding the tumour characteristics in our study, univariate analysis revealed that bilobar tumours, multifocal nodules and larger tumour size were predictive factors for decompensation, but on multivariate analysis, tumour size was the only independent predictive factor. BCLC stage was not among our independent predictive factors, possibly because decompensation was more dependent on tumour burden and serum albumin. Surprisingly, vascular invasion and extrahepatic spread were not among the predictive factors for decompensation, although they indicate a more advanced stage. This could be explained by the fact that in our study only a small number of patients had either a vascular invasion or an extrahepatic spread. In summary, pretreatment evaluation of liver function and proper staging of the tumour are of fundamental importance prior to TACE. Proper selection of patients is essential for a better outcome and lower risk of hepatic decompensation. Serum albumin and tumour burden (tumour size and $\alpha$-fetoprotein) were the independent predictors of decompensation after TACE. Future studies are warranted on a larger number of patients with a lengthier follow-up to assess these and additional factors that may predict poorer outcomes.

Acknowledgements The authors wish to thank Sameera Ezzat and Wessam Saber, Ph.D, for statistical analysis and Lark Lands, Ph.D, for her invaluable assistance in the preparation of the manuscript.

Contributors MASK and HT made substantial contributions to the conception and design of the research, acquisition of data, drafting of the article and a critical revision of the article for important intellectual content, as well as giving final approval of the version to be published. MIAZ and ME-W made substantial contributions to the conception and design of the research and acquisition of data and revised the article critically for important intellectual content, as well as giving final approval of the version to be published. RGG made substantial contributions to the conception and design of the research, analysis and interpretation of data and revised the article critically for important intellectual content, as well as giving final approval of the version to be published.

Funding This study was supported by the Hepatology and Interventional Radiology Departments at the National Liver Institute, Menoufiya University, Egypt.

Competing interests None declared.

Ethics approval Institutional Review Board of the National Liver Institute in Egypt.

Provenance and peer review Not commissioned; externally peer reviewed.

Data sharing statement No additional data are available.

Open Access This is an Open Access article distributed in accordance with the Creative Commons Attribution Non Commercial (CC BY-NC 4.0) license, which permits others to distribute, remix, adapt, build upon this work noncommercially, and license their derivative works on different terms, provided the original work is properly cited and the use is non-commercial. See: http:// creativecommons.org/licenses/by-nc/4.0/

\section{REFERENCES}

1. Shi M, Chen JA, Lin XJ, et al. Transarterial chemoembolization as initial treatment for unresectable hepatocellular carcinoma in southern China. World J Gastroenterol 2010;16:264-9.

2. Sacco R, Bertini M, Petruzzi P, et al. Clinical impact of selective transarterial chemoembolization on hepatocellular carcinoma: a cohort study. World J Gastroenterol 2009;15:1843-8.

3. Omata M, Lesmana LA, Tateishi R, et al. Asian Pacific Association for the Study of the Liver consensus recommendations on hepatocellular carcinoma. Hepatol Int 2010;4:439-74. 
4. Rampone B, Schiavone B, Confuorto G. Current management of hepatocellular cancer. Curr Oncol Rep 2010;12:186-92.

5. Bruix J, Sherman M, American Association for the Study of Liver Diseases. Management of hepatocellular carcinoma: an update. Hepatology 2011;53:1020-2.

6. Liapi E, Geschwind JF. Transcatheter and ablative therapeutic approaches for solid malignancies. J Clin Oncol 2007;25:978-86.

7. Bruix J, Sherman M, Llovet JM, et al. Clinical management of hepatocellular carcinoma. Conclusions of the Barcelona-2000 EASL conference. European Association for the Study of the Liver. $J$ Hepatol 2001;35:421-30.

8. Kothary N, Weintraub JL, Susman J, et al. Transarterial chemoembolization for primary hepatocellular carcinoma in patients at high risk. J Vasc Interv Radiol 2007:18:1517-26.

9. Vogl TJ, Naguib NN, Nour-Eldin NE, et al. Review on transarterial chemoembolization in hepatocellular carcinoma: palliative, combined, neoadjuvant, bridging, and symptomatic indications. Eur J Radiol 2009;72:505-16.

10. Chan $\mathrm{AO}$, Yuen MF, Hui CK, et al. A prospective study regarding the complications of transcatheter intraarterial lipiodo chemoembolization in patients with hepatocellular carcinoma. Cancer 2002;94:1747-52.

11. Takayasu K, Arii S, Ikai I, et al. Prospective cohort study of transarterial chemoembolization for unresectable hepatocellular carcinoma in 8510 patients. Gastroenterology 2006;131:461-9.

12. Sun Z, Li G, Ai X, et al. Hepatic and biliary damage after transarterial chemoembolization for malignant hepatic tumors: incidence, diagnosis, treatment, outcome and mechanism. Crit Rev Oncol Hematol 2011;79:164-74

13. Malagari K, Chatzimichael K, Alexopoulou E, et al. Transarteria chemoembolization of unresectable hepatocellular carcinoma with drug eluting beads: results of an open-label study of 62 patients. Cardiovasc Intervent Radiol 2008;31:269-80.

14. Buijs M, Vossen JA, Frangakis C, et al. Nonresectable hepatocellular carcinoma: long-term toxicity in patients treated with transarterial chemoembolization-single-center experience. Radiology 2008;249:346-54

15. Wigmore SJ, Redhead DN, Thomson BN, et al. Postchemoembolisation syndrome - tumour necrosis or hepatocyte injury? Br J Cancer 2003;89:1423-7.

16. Trotti A, Colevas AD, Setser A, et al. CTCAE v3.0: development of a comprehensive grading system for the adverse effects of cancer treatment. Semin Radiat Oncol 2003;13:176-81.

17. Raoul JL, Sangro B, Forner A, et al. Evolving strategies for the management of intermediate-stage hepatocellular carcinoma available evidence and expert opinion on the use of transarterial chemoembolization. Cancer Treat Rev 2011;37:212-20.

18. Liaw YF, Lin DY. Transcatheter hepatic arterial embolization in the treatment of hepatocellular carcinoma. Hepato-Gastroenterol 1990;37:484-8

19. Bronowicki JP, Vetter D, Dumas F, et al. Transcatheter oily chemoembolization for hepatocellular carcinoma. A 4- year study of 127 French patients. Cancer 1994;74:16-24.

20. Marelli L, Stigliano R, Triantos C, et al. Transarterial therapy for hepatocellular carcinoma: which technique is more effective? A systematic review of cohort and randomized studies. Cardiovasc Intervent Radiol 2007;30:6-25.

21. Pentecost MJ, Daniels JR, Teitelbaum GP, et al. Hepatic chemoembolization: safety with portal vein thrombosis. $J$ Vasc Interv Radiol 1993:4:347-51

22. Fan J, Wu ZQ, Tang ZY, et al. Multimodality treatment in hepatocellular carcinoma patients with tumor thrombi in portal vein World J Gastroenterol 2001;7:28-32.

23. Georgiades CS, Hong K, D'Angelo M, et al. Safety and efficacy of transarterial chemoembolization in patients with unresectable hepatocellular carcinoma and portal vein thrombosis. J Vasc Interv Radiol 2005;16:1653-9.

24. Herber SC, Otto G, Schneider J, et al. Transarterial chemoembolization in patients not eligible for liver transplantation: single-center results. Am J Roentgenol 2008;190:1035-42.

25. Herber S, Pitton M, Monch C, et al. [Transarterial chemoembolization (TACE) of the hepatocellular carcinoma (HCC) in patients with portal vein thrombosis-experiences]. Zentralbl Chir 2007;132:306-15.
26. Chung GE, Lee JH, Kim HY, et al. Transarterial chemoembolization can be safely performed in patients with hepatocellular carcinoma invading the main portal vein and may improve the overall survival. Radiology 2011;258:627-34.

27. Whang-Peng J, Cheng AL, Hsu C, et al. Clinical development and future direction for the treatment of hepatocellular carcinoma. $J$ Exp Clin Med 2010;2:93-103.

28. Poon RT, Fan ST, Tsang FH, et al. Locoregional therapies for hepatocellular carcinoma: a critical review from the surgeon's perspective. Ann Surg 2002;235:466-86.

29. Katsushima S, Oi H, Nakagawa K, et al. Hepatic neoplasms: effects of transcatheter arterial embolization on coagulation and fibrinolysis. Radiology 1990;174:747-50.

30. Feun LG, Reddy KR, Yrizarry JM, et al. A phase I study of chemoembolization with cisplatin and lipiodol for primary and metastatic liver cancer. Am J Clin Oncol 1994;17:405-10.

31. Rose DM, Chapman WC, Brockenbrough AT, et al. Transcatheter arterial chemoembolization as primary treatment for hepatocellular carcinoma. Am J Surg 1999;177:405-10.

32. Munk PL, Ho SGF, Legiehn GM, et al. Chemoembolisation of hepatic tumours: changes in platelet count, haemoglobin, and creatinine postembolisation. J HK Coll Radiol 2002;5:14-18.

33. Khan KN, Nakata K, Kusumoto $\mathrm{Y}$, et al. Evaluation of nontumorous tissue damage by transcatheter arterial embolization for hepatocellular carcinoma. Cancer Res 1991;51:5667-71.

34. Gu YK, Luo RG, Huang JH, et al. Transarterial embolization ablation of hepatocellular carcinoma with a lipiodol-ethanol mixture. World $J$ Gastroenterol 2010;16:5766-72.

35. Shin SW. The current practice of transarterial chemoembolization for the treatment of hepatocellular carcinoma. Korean $\mathrm{J}$ Radiol 2009;10:425-34.

36. Hucke F, Pinter M, Graziadei I, et al. How to STATE suitability and START transarterial chemoembolization in patients with intermediate stage hepatocellular carcinoma. $J$ Hepatol 2014;61:1287-96.

37. Kadalayil L, Benini R, Pallan L, et al. A simple prognostic scoring system for patients receiving transarterial embolisation for hepatocellular cancer. Ann Oncol 2013;24:2565-70.

38. Nomura F, Ohnishi K, Tanabe Y. Clinical features and prognosis of hepatocellular carcinoma with reference to serum alpha-fetoprotein levels. Analysis of 606 patients. Cancer 1989;64:1700-7.

39. Ikeda M, Okada S, Yamamoto S, et al. Prognostic factors in patients with hepatocellular carcinoma treated by transcatheter arterial embolization. Jpn J Clin Oncol 2002;32:455-60.

40. Matsui O, Kadoya M, Yoshikawa J, et al. Subsegmental transcatheter arterial embolization for small hepatocellular carcinomas: local therapeutic effect and 5-year survival rate. Cancer ChemotherPharmacol 1994;33(Suppl):S84-8

41. Camma C, Schepis F, Orlando A, et al. Transarterial chemoembolization for unresectable hepatocellular carcinoma meta-analysis of randomized controlled trials. Radiology 2002;224:47-54.

42. Llado L, Virgili J, Figueras J, et al. A prognostic index of the survival of patients with unresectable hepatocellular carcinoma after transcatheter arterial chemoembolization. Cancer 2000;88:50-7.

43. Okuda K, Ohtsuki T, Obata H, et al. Natural history of hepatocellular carcinoma and prognosis in relation to treatment. Study of 850 patients. Cancer 1985;56:918-28.

44. Ueno K, Miyazono N, Inoue $\mathrm{H}$, et al. Transcatheter arterial chemoembolization therapy using iodized oil for patients with unresectable hepatocellular carcinoma: evaluation of three kinds of regimens and analysis of prognostic factors. Cancer 2000;88:1574-81.

45. Shah SR, Riordan SM, Karani J, et al. Tumour ablation and hepatic decompensation rates in multi-agent chemoembolization of hepatocellular carcinoma. QJM 1998;91:821-8.

46. Hwang JI, Chow WK, Hung SW, et al. Development of a safety index of transarterial chemoembolization for hepatocellular carcinoma to prevent acute liver damage. Anticancer Res 2005;25:2551-4.

47. Yip WM, Hung HG, Lok KH, et al. Outcome of inoperable hepatocellular carcinoma patients receiving transarterial chemoembolisation: a real-life retrospective analysis in a Hong Kong regional hospital. Hong Kong Med J 2009;15:339-45. 